\title{
The Nucleotide Oligomerization Domain-Like Receptors in Kidney Injury
}

\author{
Xiaojie Wang Fan Yi \\ Department of Pharmacology, Shandong University School of Medicine, Jinan, PR China
}

\author{
Key Words \\ Inflammation · Kidney disease · NLRP3 - NOD2 - Pattern \\ recognition receptors
}

\begin{abstract}
Background: Inflammation is a hallmark of almost all forms of renal injury and the activation of the innate immune system is of importance in the development of many kidney diseases. Pattern recognition receptors (PRRs) act as sensors of the innate immune system to detect pathogen- or damage-associated molecular patterns, which initiate immune responses to resolve infections and repair damaged tissues. Abnormalities in PRR activation will lead to excessive inflammation. Summary: Nucleotide oligomerization domain (NOD)-like receptors (NLRs) are recently identified intracellular PRRs that are essential to innate immune responses and tissue homeostasis. A better understanding of the function of NLRs will provide unexpected opportunities to develop new therapies for kidney diseases by modulation of the innate immune system. Key Messages: NLRs are constitutively expressed in the kidney and emerging evidence has shown that activation of NLRs plays an important role in the pathogenesis of renal injury. Among NLRs, NOD2 and NLRP3 inflammasome are the best characterized members in the kidney. In this review, we summarize current knowledge about the pathological mechanisms that are related to NOD2 and
\end{abstract}

NLRP3 inflammasome in various kidney diseases by their canonical and non-canonical effects and discuss the opportunities of pharmacological targeting of NLR-mediated signaling pathways at multiple levels for the treatment of renal disease.

(c) 2016 S. Karger AG, Basel

\section{Introduction}

Emerging evidence has implicated that the activation of innate immune signaling and inflammatory responses are of importance in the pathogenesis of renal disease. In contrast to the adaptive immune system, the innate immune system plays a key role as a first response to pathogens or tissue injury [1-3], which is initiated by pattern recognition receptors (PRRs) in the recognition of pathogen-associated molecular patterns (PAMPs) and damage-associated molecular patterns (DAMPs) that are independent of the clonal expansion of antigen-specific lymphocytes [4]. Although the activation of PRRs can induce the recruitment of immune cells, initiate tissue repair and activate adaptive immune responses, inappropriate or chronic PRR activation results in excessive inflammation and exacerbates tissue damage [5-7]. PRRs are classified into several subfamilies, which are composed of C-type lectin-like receptors, Toll-like receptors,

\section{KARGER}

E-Mail karger@karger.com

www.karger.com/kdd
(C) 2016 S. Karger AG, Basel

2296-9381/16/0021-0028\$39.50/0
Fan $\mathrm{Yi}, \mathrm{PhD}$

Department of Pharmacology

Shandong University School of Medicine

\#44 Wenhua Xi Road, Jinan, Shandong 250012 (PR China)

E-Mail fanyi@sdu.edu.cn 


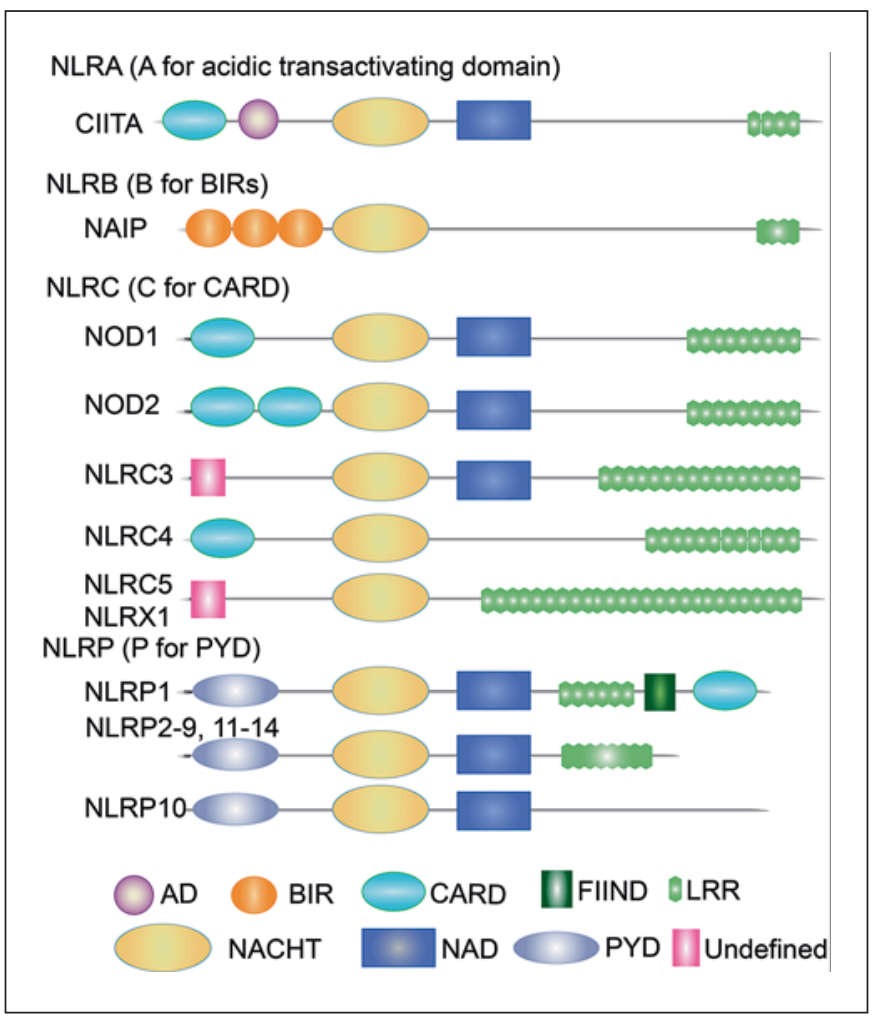

Fig. 1. Classification and molecular structures of NLRs. AD = Activator domain; BIR = baculovirus inhibitor of an apoptosis protein repeat; FIIND = function to find domain; LRR = leucine-rich repeat; $\mathrm{NACHT}=\underline{\mathrm{NAIP}}$ (neuronal apoptosis inhibitor protein), C2TA (MHC class 2 transcription activator), $\underline{H E T-E}$ (incompatibility locus protein from Podospora anserina) and TP1 (telomerase-associated protein); NAD = NACHT-associated domain.

nucleotide oligomerization domain (NOD)-like receptors (NLRs), retinoic acid inducible gene I-like receptors and absent in melanoma 2-like receptors [8]. Among them, NLRs are recently identified intracellular PRRs [9, $10]$ and 22 members have been identified in humans and 34 in mice which contain a highly conserved nucleotide binding and oligomerization (NACHT) domain that is required for self-oligomerization of the proteins upon ligand recognition, a C-terminal leucine-rich repeat that acts as a sensing domain, and an $\mathrm{N}$-terminal effector domain that mediates downstream signaling such as caspase activation and recruitment domain (CARD) or a pyrin domain (PYD) [11] as shown in figure 1. Among them, NOD1/2 can activate NF- $\kappa B$ and MAPK signaling pathways in response to peptidoglycan-related molecules [12], resulting in the transcription of a large number of proinflammatory factors and mediating inflammatory processes to establish an appropriate immune response

NOD-Like Receptors and Renal Function
[13]. Another important class of the NLR family, such as NLRP1, NLRP3, NLRP6 or NLRP12, can oligomerize to form an inflammasome with the PYD-CARD adaptor protein ASC (apoptosis-associated speck-like protein containing a CARD) and caspase- 1 , which are associated with a wide range of physiological and pathological processes by activation of caspase-1 [14]. Activation of caspase- 1 promotes the processing and secretion of IL- $1 \beta$ and IL-18 contributing to the inflammatory response [15]. NLRs are not only expressed in immunocytes, but also widely distributed in various renal parenchymal cells, which induce innate immune responses by the recognition of exogenous PAMPs or endogenous DAMPs. A growing number of studies have demonstrated that NLRs are involved in the pathogenesis of various kidney diseases [16-20]. Among the NLRs, NOD2 and NLRP3 inflammasome are the best characterized members in the kidney. In this review, we focus on NOD2 (fig. 2) and NLRP3 (fig. 3) and their canonical and non-canonical effects in acute kidney injury (AKI) and chronic kidney disease $(\mathrm{CKD})$ and discuss the opportunities of targeting of NLR-mediated signaling pathways at multiple levels for the treatment of renal disease.

\section{NLRs and AKI}

AKI is a common complication associated with high morbidity and mortality, which is often induced by ischemia/reperfusion injury (IRI), sepsis or toxins. AKI involves renal inflammation related to the activation of innate immunity. NLRs are constitutively expressed in the kidney and emerging evidence has shown that the activation of NLRs plays an important role in the pathogenesis of AKI [21].

\section{Renal IRI}

One of the most common cause of AKI is renal IRI, which mainly attributes the organ damage to the inflammatory and oxidative stress responses induced by renal ischemia and subsequent reperfusion. Numerous studies have indicated that inflammatory processes engaging both innate and adaptive immune responses are responsible for the initial renal injury such as tubular apoptosis or necrosis and mediate long-term structural changes including interstitial fibrosis or repair $[22,23]$. Shigeoka et al. [24] for the first time reported that NOD1 and NOD2 were present in renal tubular epithelial cells, and that gene deficiency of NOD1 or NOD2 protected against renal IRI and the simultaneous deficiency of NOD1 and NOD2 
Fig. 2. Summarized recent findings on the role of NOD2 and its canonical and noncanonical effects in kidney injury.
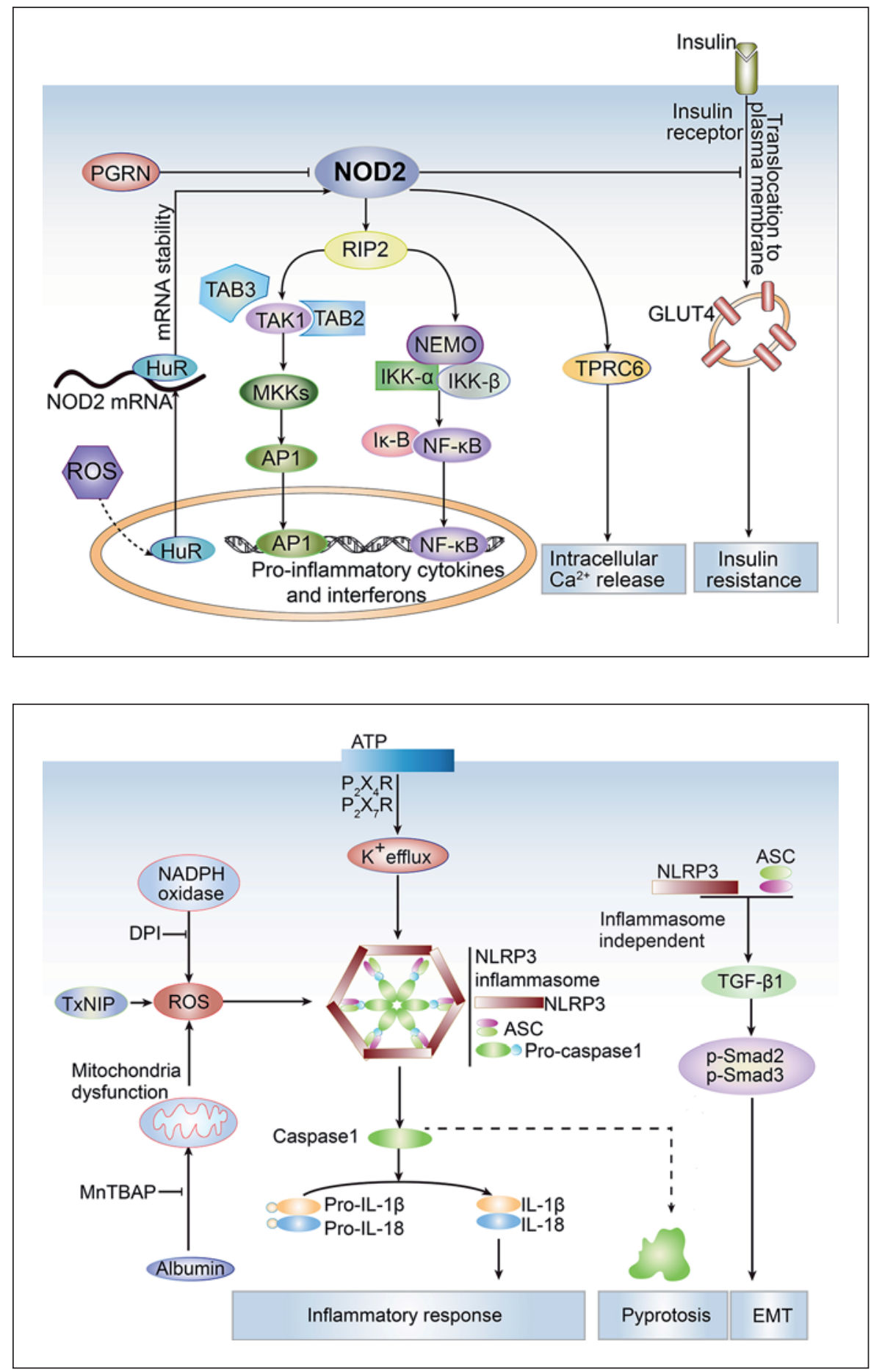

Fig. 3. Summarized recent findings on the function, activation and canonical and non-canonical effects of NLRP3 in kidney injury. EMT = Epithelial-mesenchymal transition. and treatment of mice with recombinant PGRN resulted in a dramatic reduction in renal dysfunction. Interestingly, we observed an increase in NOD2 expression after renal IRI and a negative correlation between PGRN and NOD2 expression in the kidney. Considering that NOD2 
initiates inflammatory responses largely dependent on $\mathrm{NF}-\kappa \mathrm{B}$ activation [25], we further detected the effect of PGRN on NF- $\kappa B$ and found that NOD2-dependent activation of NF- $\kappa B$ was also negatively regulated by PGRN. Together, these results suggest that PGRN may be an innovative therapeutic strategy for treating patients with AKI by targeting NOD2-mediated inflammatory responses [23].

NLRP3 has been demonstrated to contribute to renal IRI $[26,27]$. It was found that NLRP 3 and adaptor protein ASC were highly expressed in renal tubular epithelium, and the absence of NLRP3, but not ASC or the downstream inflammasome targets, protected from renal IRI, indicating that NLRP3 contributes to renal IRI by a direct effect on renal tubular epithelium, which is independent of inflammasome-induced proinflammatory cytokine production [26]. In addition, a very recent study by Bakker et al. [28] explored the role of NLRP3 in the repair phase of renal ischemia/reperfusion and investigated the contribution of leukocyte- versus renal-associated NLRP3 by studying bone marrow chimeric mice. It was found that although both renal- and leukocyte-associated NLRP3 were detrimental to renal function after renal IRI, this was through different mechanisms. Leukocyte-associated NLRP3 was associated with increased tubular epithelial apoptosis, whereas renal-associated NLRP3 impaired the tubular epithelial repair response, suggesting NLRP3 as a negative regulator of resident tubular cell proliferation in addition to its detrimental role in renal fibrosis and inflammation.

\section{Toxin-Induced AKI}

The kidney is susceptible to toxin injury. Cisplatin is a major course of toxin-induced AKI, which triggers the apoptosis of renal tubular epithelial cells followed by inflammation and fibrosis. In our previous studies, we found that PGRN protected against cisplatin-induced AKI, which was also associated with NOD2-mediated signaling (unpublished data). Interestingly, Kim et al. [27] provided evidence showing that NLRP3-deficient mice were protected against ischemic but not cisplatin-induced AKI. They found that although in the kidneys from mice with cisplatin-induced AKI, inflammasome components ASC and caspase- 1 was upregulated, the increase in caspase- 1 in kidney and proximal tubules was not associated with an increase in NLRP3 protein. Furthermore, they noted that NLRP3-deficient mice were not protected against cisplatin-induced AKI, despite previous studies having demonstrated that caspase- 1 is a mediator of cisplatin-induced AKI.

NOD-Like Receptors and Renal Function

\section{Sepsis-Induced AKI}

$\mathrm{AKI}$ is one of the most important factors determining morbidity and mortality in the prognosis of sepsis. Unlike the pathogenic mechanisms of other causes of AKI, sepsis is associated with an entire orchestra of cellular mechanisms, which potentiate each other and ultimately induce clinical AKI. The microcirculation is the important physiological compartment where these mechanisms exert their integrated and deleterious action [29]. Although the role of NLRs in renal injury in response to sepsis is not well known, emerging studies have reported the participation of NLRs in sepsis-induced AKI. A study from Cao et al. [30] investigated the role of NLRP3 inflammasomes in renal injury in a cecal ligation and puncture model of sepsis-induced AKI. It was found that cecal ligation and puncture upregulated NLRP3, ASC and caspase-1 expression and caspase- 1 activation in the kidney and that genetic deletion of NLRP3 reversed the cecal ligation and puncture-induced increases in creatinine and neutrophil infiltration. Moreover, very recent studies have also reported that mangiferin [31] and exogenous carbon monoxide [32] attenuated sepsis-induced AKI via inhibition of NLRP3 inflammasome activation.

\section{NLRs and CKD}

Inflammation is a hallmark of almost all forms of CKD and the innate immune system participates in many inflammatory processes during the development of CKD. Several PRRs have been identified as contributing to the development of a CKD-specific proinflammatory microenvironment. In tissue from human renal biopsies, a wide variety of CKD exhibited increased expression of NOD2 and NLRP3 mRNA, including IgA nephropathy, focal segmental glomerulosclerosis, lupus nephritis, minimal change disease and diabetic nephropathy, which correlated with renal function $[21,33]$, strongly supporting the role for NLRs in CKD. In this section, we review the current knowledge regarding NOD2 and NLRP3 inflammasome signaling and outline existing evidence on their functional roles in CKD.

\section{Renal Interstitial Fibrosis}

Progression of renal injury ultimately leads to renal interstitial fibrosis, which is characterized by activation and proliferation of renal interstitial fibroblasts as well as accumulation of extracellular matrix components. During the development of renal fibrosis, inflammatory responses are induced and involved in this process [34]. 
The unilateral ureteral obstruction model is usually employed to explore the mechanisms of renal fibrosis. Compared with wild-type mice, NLRP3-deficient mice had less tubular injury, inflammation and fibrosis after unilateral ureteral obstruction, accompanied by a reduction in caspase- 1 activation and secretion of IL- $1 \beta$ and IL- 18 . Furthermore, bone marrow chimeras revealed that NLRP3 mediated the injurious and inflammatory processes in both immune and non-immune cellular compartments [33]. Additionally, Wang et al. [20] also reported that NLRP3 promoted TGF- $\beta$ signaling and Smad activation in epithelial cells and that the effect of NLRP 3 on TGF- $\beta 1$ signaling was independent of the inflammasome components. They found that NLRP3 expression was increased in response to TGF- $\beta 1$ and associated with epithelial-mesenchymal transition. Furthermore they demonstrated that TGF- $\beta$ stimulation not only increased NLRP3 expression in a Smad3-dependent manner, but that NLRP3 and ASC were required for TGF- $\beta$-mediated Smad 2 and Smad3 phosphorylation, supporting a novel role for NLRP3 in promoting TGF- $\beta$ signaling and Smad activation in epithelial cells independent of the inflammasome [20]. On the contrary, Pulskens et al. [35] reported the non-canonical effects of NLRP3 following progressive renal injury induced by unilateral ureteral obstruction. They found that the deficiency of NLRP3 led to early tubular damage and interstitial edema, suggesting a protective role of NLRP3 against progressive renal injury by preserving the vascular barrier and epithelial integrity in the kidneys.

In fact, proteinuria is not only a common feature of $\mathrm{CKD}$, but also an independent causative factor for the development of tubulointerstitial inflammation and fibrosis [36, 37]. Recent studies have indicated that the NLRP3 inflammasome contributes to albumin-induced lesions in tubular cells [38, 39]. Liu et al. [40] demonstrated that endocytic receptor-lysosome-mediated albumin reabsorption was involved in the tubular cell activation of NLRP3 inflammasome and tubulointerstitial inflammation. Moreover, studies from Zhuang et al. reported that NLRP3 inflammasome mediated albumininduced renal tubular injury through impaired mitochondrial function [41] and further provided direct evidence indicating the important role of the albuminNLRP3 inflammasome axis in mediating the impairment of renal tubular tight junctions and integrity [42]. Additionally, Fang et al. [43] also provided evidence showing the involvement of endoplasmic reticulum stress in albuminuria-induced inflammasome activation in renal proximal tubular cells.

\section{Diabetic Nephropathy}

Diabetic nephropathy is one of the major microvascular complications of diabetes mellitus and the most common cause of end-stage renal disease. Dysregulation of the innate immune response via NLRs has been implicated in the development and progression of diabetic nephropathy [4]. We have identified the upregulation of NOD2 in both renal parenchymal cells and infiltrating immune cells from renal biopsies of diabetic patients and high-fat diet/streptozotocin-induced diabetic mice. We further found that NOD2 deficiency ameliorated diabetic renal injury. Evidence for a functional contribution of NOD2 expression to renal function came from an in vitro study showing that NOD2 activation induced ERK1 and ERK2, JNK and p38 MAPK signaling as well as NF$\kappa \mathrm{B}$, which was associated with high glucose-induced podocyte inflammatory responses and apoptosis. Interestingly, we also found that NOD2 activation induced podocyte insulin resistance, impaired insulin-induced glucose uptake and reduced nephrin expression in podocytes [21]. These results provided direct evidence on the contribution of NOD2 canonical and non-canonical effects in diabetic nephropathy [44]. Furthermore, we found that human antigen $\mathrm{R}$ (HuR), a RNA-binding protein which regulates mRNA cargos that contain adenylate-uridylate-rich elements (AREs) in the $3^{\prime}$-untranslated region [45], contributed to NOD2 mRNA stability in diabetic nephropathy [46]. Among four AUUUA regions (ARE1-4) of the $3^{\prime}$-untranslated region of NOD2, HuR can directly bind to ARE4 of NOD2 to enhance NOD2 mRNA stability and expression. Regarding the regulation of HuR activity in the kidney, NADPH oxidase-mediated oxidative stress has been reported to be associated with $\mathrm{HuR}$ translocation and activity [47, 48]. NOX4 is an important subunit of NADPH oxidase to produce renal reactive oxygen species (ROS) and deficiency of NOX4 results in renal protection from glomerular injury in diabetic mice $[49,50]$. We further found that NOX4-mediated redox signaling contributed to the expression and translocation of HuR and NOD2 mRNA stability [46].

In addition to NOD2, NLRP3 inflammasome-mediated inflammatory responses have also been implicated in diabetic nephropathy. In patients with type 2 diabetes-associated nephropathy, NLRP3 expression was significantly increased in renal tubular epithelial cells, which positively correlated with urinary IL- $1 \beta$ and IL18 levels [51]. Wang et al. [52] reported that the NLRP3 inflammasome was activated in the kidney of streptozotocin-induced diabetic rats and that suppression of 
NLRP3 inflammasome activation significantly reduced renal inflammation and improved renal function. Bakker et al. [53] further demonstrated that NLRP3 plays a key role in diet-induced nephropathy and renal cholesterol accumulation. It was found that NLRP3 deficiency ameliorated fructose-induced renal injury by reduced renal inflammation, fibrosis, albuminuria and hyperuricemia. Mechanically, under diabetic conditions, NLRP3 was activated by ROS or extracellular ATP [54]. Hyperglycemia induced the expression of the ATP receptor P2X4 in renal tubular epithelial cells of patients with type 2 diabetic nephropathy, which correlated with IL-1 cytokine release. ATP-P2X4 signaling was further found to mediate high glucose-induced activation of the NLRP3 inflammasome and to regulate IL-1 family cytokine secretion, resulting in tubulointerstitial inflammation [51]. Regarding the regulation of NLRP3 inflammasome by ROS, Gao et al. [55, 56] recently found that hyperglycemia-induced NADPH oxidase activation was driven by thioredoxin-interacting protein (TXNIP) which subsequently triggered NLRP3 inflammasome activation in podocytes and ultimately led to podocyte injury. A recent study also indicated that thrombomodulin domain 1 ameliorated diabetic nephropathy in mice via anti-NF- $\mathrm{B} / \mathrm{NLRP} 3$ inflammasome-mediated inflammatory responses, enhancement of NRF2 antioxidant activity and inhibition of apoptosis [57].

More interestingly, although it is generally accepted that hyperuricemia-induced NLRP3 activation of macrophages contributes to the progression of diabetic nephropathy [58], recent studies have unraveled the role of the activated NLRP3 inflammasome in glomerular resident cells and addressed the importance of NLRP3 inflammasome activation in non-myeloid-derived cells in diabetic nephropathy $[59,60]$. It was found that abolishing NLRP3 or caspase-1 expression in bone marrow-derived cells failed to protect mice against diabetic nephropathy, as evidenced by the fact that albuminuria and mesangial expansion in $\mathrm{db} / \mathrm{db}$ mice transplanted with $\mathrm{NLRP3}^{-/-}$or caspase- $1^{-/-}$bone marrow increased to the same extent as those in control $\mathrm{db} / \mathrm{db}$ mice. Conversely, $\mathrm{NLRP}^{-/-}$mice were protected against diabetic nephropathy despite transplantation of wild-type bone marrow, indicating that the NLRP3 in renal resident cells significantly contributed to the pathogenesis of diabetic nephropathy. Further studies are required to use cell-specific deletion of the NLRP3 inflammasome to explore the role of inflammasome activation in glomerular and tubular epithelial cells individually.

\section{Hyperhomocysteinemia-Induced Renal Injury}

Hyperhomocysteinemia is one major metabolic disorder of amino acids, which is a medical condition characterized by an abnormally high level of homocysteine in the blood, conventionally described as $>10 \mu \mathrm{mol} / \mathrm{l}$. Hyperhomocysteinemia is considered an important independent risk factor in the progression of end-stage renal disease and in the development of cardiovascular complications related to end-stage renal disease $[61,62]$. In our study, we found that NOD2 deficiency ameliorated renal injury in mice with hyperhomocysteinemia. We further discovered the non-canonical effects of NOD2 in mediating $\mathrm{Ca}^{2+}$ signaling showing that NOD2 regulated transient receptor potential cation channel 6 expression and activity via nephrin, resulting in intracellular $\mathrm{Ca}^{2+}$ release and podocyte cytoskeleton rearrangement and apoptosis. These results indicate that NOD2-mediated $\mathrm{Ca}^{2+}$ signaling is one of the critical signal transduction pathways that links innate immunity mediator NOD2 to homocysteine-induced podocyte injury. In experimental mice with hyperhomocysteinemia, Zhang et al. [63] found that NLRP3 inflammasome formation and activation in glomerular podocytes were detected at an early stage and that homocysteineassociated albuminuria, foot process effacement of podocytes, loss of podocyte slit diaphragm molecules and glomerulosclerosis at the late stage were significantly improved by inhibition of local ASC or caspase-1, indicating that NLRP3 inflammasome activation is an important molecular mechanism triggering podocyte injury and ultimately resulting in glomerulosclerosis. Further studies demonstrated that NADPH oxidasemediated oxidative stress was importantly involved in the switching on NLRP3 inflammasomes in podocytes, which led to the downstream recruitment of immune cells, ultimately resulting in glomerular injury [64]. An important factor links changes in oxidative stress to NLRP3 activation is TXNIP, a negative regulator of the antioxidant thioredoxin. When ROS accumulates, TXNIP can sense ROS and time-dependently dissociate from thioredoxin to bind with NLRP3, leading to inflammasome formation and activation. Abais et al. [65] reported that inhibition of TXNIP prevented homocysteine-induced TXNIP protein recruitment to form NLRP3 inflammasomes and reduced caspase- 1 activity in glomeruli of mice with hyperhomocysteinemia, indicating that TXNIP binding to NLRP3 is a key signaling mechanism for homocysteine-induced NLRP3 inflammasome activation. 


\section{Renal Injury Associated with Hypertension}

Hypertension is now considered a chronic, low-grade inflammatory disease, with the kidney representing a major site of this inflammation. Although studies on NLRs in renal injury associated with hypertension are very limited, a very recent study by Krishnan et al. [66] highlights the crucial role of inflammasome activity in one kidney/deoxycorticosterone acetate/salt model of hypertension in mice. They found that deoxycorticosterone acetate/salt-induced hypertension in mice was associated with increased renal expression of NLRP3, ASC, caspase- 1 and IL- $1 \beta$ and further demonstrated that renal inflammation and fibrosis in this model were dependent on inflammasome activity.

\section{Crystalline Nephropathies}

Crystals are particles of endogenous inorganic or organic composition that can trigger renal injury when deposited or formed inside the kidney [67]. NLRP3 inflammasome-mediated IL- $1 \beta$ secretion is recognized to be the essential pathophysiological element of crystal- and particle-induced inflammation and has been demonstrated to contribute to crystalline nephropathies. Mulay et al. [68] observed that calcium oxalate crystals induced renal inflammation by NLRP3. They found that calcium oxalate crystals triggered IL- $1 \beta$-dependent innate immunity via the NLRP3/ASC/caspase-1 axis in intrarenal mononuclear phagocytes and directly damaged tubular cells, leading to the release of the NLRP3 agonist ATP. Consistently, in an animal model of progressive oxalate nephropathy by feeding mice a diet high in soluble oxalate, NLRP3 expression was significantly upregulated in the kidney and NLRP3-deficient mice were completely protected from the progressive renal failure and death that occurred in wild-type mice fed the diet high in soluble oxalate, which was associated with NLRP3-mediated inflammation rather than oxalate homeostasis by intestinal handling [69]. Further studies are needed to clarify whether all crystalline nephropathies such as adenine or urate nephropathy share the same mechanisms.

\section{Summary and Future Perspectives}

The NLR family is becoming increasingly recognized as integral to the pathogenesis of many renal diseases and their complications. This review highlights the current findings on NOD2- and NLRP3-mediated canonical and non-canonical signaling pathways in AKI and CKD (fig. 2, 3). Despite remarkable progress in NLRs, there are still numerous aspects that need to be understood in the kid-

ney. First of all, in the kidney, the molecular mechanisms for individual PRRs to induce pleiotropic outcomes remain largely unknown. In addition, controversies exist on the certainty of detrimental or beneficial effects of some NLRs in different disease states or different experimental animal models. For example, a recent study indicates a protective role of NLRP3 against progressive renal injury by preventing early renal interstitial edema and vascular permeability in unilateral ureteral obstruction [35]. Second, much of our knowledge of NLRs is limited to experimental models, and it is thus necessary to elucidate their roles in patients under clinical investigation. Third, novel and selective NLR inhibitors are urgently needed. Although scientific progress in the field of NOD inhibitors, including the recently reported selective inhibitors of NOD1 and NOD2, has been made [70], the therapeutic potential of pharmacological modulation of NOD1 and NOD2 signaling needs to be further investigated. In addition, strategies for targeting the NLRP3 inflammasome pathway have been limited to inhibitors of IL-1 $\beta$, specific inflammasome inhibitors are not yet available. Collectively, a better understanding of the function of NLRs and the development of NLR inhibitors will provide unexpected opportunities to develop new therapies for kidney disease by modulation of the innate immune system.

\section{Acknowledgements}

This study was supported by the National 973 Basic Research Program of China (2012CB517700), the National Science Fund for Distinguished Young Scholars to F. Yi (81525005), the National Nature Science Foundation of China (81371317, 81328006 and 81470958), the Program for Changjiang Scholars and Innovative Research Team in University (PCSIRT, IRT13028) and the Fundamental Research Funds of Shandong University (2014JC027).

\section{Conflict of Interest Statement}

All authors declare that they have no competing interests.

References

Kidney Dis 2016;2:28-36 DOI: $10.1159 / 000444736$

\footnotetext{
1 Vande Walle L, Van Opdenbosch N, Jacques $P$, et al: Negative regulation of the NLRP3 inflammasome by A20 protects against arthritis. Nature 2014;512:69-73.

$\checkmark 2$ Heneka MT, Kummer MP, Stutz A, et al: NLRP 3 is activated in Alzheimer's disease and contributes to pathology in APP/PS1 mice. Nature 2013;493:674-678.

-3 Kayagaki N, Warming S, Lamkanfi M, et al: Non-canonical inflammasome activation targets caspase-11. Nature 2011;479:117-121.
}

Wang/Yi 
4 Wada J, Makino H: Innate immunity in dia- 22 Gluba A, Banach M, Hannam S, Mikhailidis betes and diabetic nephropathy. Nat Rev Nephrol 2016;12:13-26.

5 Bradbury J: Zebularine: a candidate for epigenetic cancer therapy. Drug Discov Today 2004;9:906-907.

6 Walsh D, McCarthy J, O’Driscoll C, Melgar S: Pattern recognition receptors - molecular orchestrators of inflammation in inflammatory bowel disease. Cytokine Growth Factor Rev 2013;24:91-104.

7 Fukata M, Vamadevan AS, Abreu MT: Tolllike receptors (TLRs) and Nod-like receptors (NLRs) in inflammatory disorders. Semin Immunol 2009;21:242-253.

$\checkmark 8$ Takeuchi O, Akira S: Pattern recognition receptors and inflammation. Cell 2010;140: 805-820.

-9 Fritz JH, Ferrero RL, Philpott DJ, Girardin SE: Nod-like proteins in immunity, inflammation and disease. Nat Immunol 2006;7:12501257.

10 Kersse K, Bertrand MJ, Lamkanfi M, Vandenabeele P: NOD-like receptors and the innate immune system: coping with danger, damage and death. Cytokine Growth Factor Rev 2011; 22:257-276.

- 11 Zewinger S, Schumann T, Fliser D, Speer T: Innate immunity in CKD-associated vascular diseases. Nephrol Dial Transplant 2015, Epub ahead of print.

12 Inohara N, Chamaillard M, McDonald C, Nuñez G: NOD-LRR proteins: role in host-microbial interactions and inflammatory disease. Annu Rev Biochem 2005;74:355-383.

13 Elinav E, Strowig T, Henao-Mejia J, Flavell RA: Regulation of the antimicrobial response by NLR proteins. Immunity 2011;34:665679.

14 Schroder K, Tschopp J: The inflammasomes. Cell 2010;140:821-832.

15 Guo H, Callaway JB, Ting JP: Inflammasomes: mechanism of action, role in disease, and therapeutics. Nat Med 2015;21:677-687.

16 Chang A, Ko K, Clark MR: The emerging role of the inflammasome in kidney diseases. Curr Opin Nephrol Hypertens 2014;23:204-210.

$\checkmark 17$ Turner CM, Arulkumaran N, Singer M, Unwin RJ, Tam FW: Is the inflammasome a potential therapeutic target in renal disease? BMC Nephrol 2014; 15:21.

-18 Benetti E, Chiazza F, Patel NS, Collino M: The NLRP3 Inflammasome as a novel player of the intercellular crosstalk in metabolic disorders. Mediators Inflamm 2013;2013:678627.

19 Hao JL, Li YF, Li RS: A novel mechanism of NALP3 inducing ischemia reperfusion injury by activating MAPK pathway in acute renal failure. Med Hypotheses 2013;80:463-465.

20 Wang W, Wang X, Chun J, et al: Inflammasome-independent NLRP3 augments TGFbeta signaling in kidney epithelium. J Immunol 2013;190:1239-1249.

21 Du P, Fan B, Han H, et al: NOD2 promotes renal injury by exacerbating inflammation and podocyte insulin resistance in diabetic nephropathy. Kidney Int 2013;84:265-276. DP, Sakowicz A, Rysz J: The role of Toll-like receptors in renal diseases. Nat Rev Nephrol 2010;6:224-235.

23 Zhou M, Tang W, Fu Y, et al: Progranulin protects against renal ischemia/reperfusion injury in mice. Kidney Int 2015;87:918-929.

24 Shigeoka AA, Kambo A, Mathison JC, et al: Nod1 and Nod2 are expressed in human and murine renal tubular epithelial cells and participate in renal ischemia reperfusion injury. J Immunol 2010;184:2297-2304

25 Ting JP, Duncan JA, Lei Y: How the noninflammasome NLRs function in the innate immune system. Science 2010;327:286-290.

26 Shigeoka AA, Mueller JL, Kambo A, et al: An inflammasome-independent role for epithelial-expressed Nlrp3 in renal ischemia-reperfusion injury. J Immunol 2010;185:6277-6285.

27 Kim HJ, Lee DW, Ravichandran K, et al: NLRP3 inflammasome knockout mice are protected against ischemic but not cisplatininduced acute kidney injury. J Pharmacol Exp Ther 2013;346:465-472.

28 Bakker PJ, Butter LM, Claessen N, et al: A tissue-specific role for Nlrp3 in tubular epithelial repair after renal ischemia/reperfusion. Am J Pathol 2014;184:2013-2022.

29 Zarbock A, Gomez H, Kellum JA: Sepsis-induced acute kidney injury revisited: pathophysiology, prevention and future therapies. Curr Opin Crit Care 2014;20:588-595.

30 Cao Y, Fei D, Chen M, et al: Role of the nucleotide-binding domain-like receptor protein 3 inflammasome in acute kidney injury. FEBS J 2015;282:3799-3807.

31 He L, Peng X, Zhu J, et al: Mangiferin attenuate sepsis-induced acute kidney injury via antioxidant and anti-inflammatory effects. Am J Nephrol 2014;40:441-450.

32 Wang P, Huang J, Li Y, et al: Exogenous carbon monoxide decreases sepsis-induced acute kidney injury and inhibits NLRP3 inflammasome activation in rats. Int J Mol Sci

33 Vilaysane A, Chun J, Seamone ME, et al: The NLRP3 inflammasome promotes renal inflammation and contributes to CKD. J Am Soc Nephrol 2010;21:1732-1744.

34 Liu N, Zhuang S: Treatment of chronic kidney diseases with histone deacetylase inhibitors. Front Physiol 2015;6:121.

35 Pulskens WP, Butter LM, Teske GJ, et al: Nlrp3 prevents early renal interstitial edema and vascular permeability in unilateral ureteral obstruction. PLoS One 2014;9:e85775.

36 Abbate M, Zoja C, Remuzzi G: How does proteinuria cause progressive renal damage? J Am Soc Nephrol 2006;17:2974-2984.

37 Eddy AA: Proteinuria and interstitial injury. Nephrol Dial Transplant 2004;19:277-281.

38 Liu D, Xu M, Ding LH, et al: Activation of the Nlrp3 inflammasome by mitochondrial reactive oxygen species: a novel mechanism of albumin-induced tubulointerstitial inflammation. Int J Biochem Cell Biol 2014;57:7-19. 2015;16:20595-20608
9 Ding LH, Liu D, Xu M, et al: Enalapril inhibits tubulointerstitial inflammation and NLRP3 inflammasome expression in BSA-overload nephropathy of rats. Acta Pharmacol Sin 2014;35:1293-1301.

40 Liu D, Wen Y, Tang TT, et al: Megalin/cubulin-lysosome-mediated albumin reabsorption is involved in the tubular cell activation of NLRP3 inflammasome and tubulointerstitial inflammation. J Biol Chem 2015;290:1801818028.

41 Zhuang Y, Ding G, Zhao M, et al: NLRP3 inflammasome mediates albumin-induced renal tubular injury through impaired mitochondrial function. J Biol Chem 2014;289: 25101-25111.

42 Zhuang Y, Hu C, Ding G, et al: Albumin impairs renal tubular tight junctions via targeting the NLRP3 inflammasome. Am J Physiol Renal Physiol 2015;308:F1012-F1019.

-43 Fang L, Xie D, Wu X, Cao H, Su W, Yang J: Involvement of endoplasmic reticulum stress in albuminuria induced inflammasome activation in renal proximal tubular cells. PLoS One 2013;8:e72344.

44 Anders HJ, Lech M: NOD-like and Toll-like receptors or inflammasomes contribute to kidney disease in a canonical and a non-canonical manner. Kidney Int 2013;84:225228

45 Brennan CM, Steitz JA: HuR and mRNA stability. Cell Mol Life Sci 2001;58:266-277.

46 Shang J, Wan Q, Wang X, et al: Identification of NOD2 as a novel target of RNA-binding protein HuR: evidence from NADPH oxidase-mediated HuR signaling in diabetic nephropathy. Free Radic Biol Med 2015;79:217227.

47 Lin FY, Chen YH, Lin YW, et al: The role of human antigen R, an RNA-binding protein, in mediating the stabilization of toll-like receptor 4 mRNA induced by endotoxin: a novel mechanism involved in vascular inflammation. Arterioscler Thromb Vasc Biol 2006;26: 2622-2629.

48 Hsu CK, Lee IT, Lin CC, Hsiao LD, Yang CM: Nox2/ROS-dependent human antigen $\mathrm{R}$ translocation contributes to TNF- $\alpha$-induced SOCS-3 expression in human tracheal smooth muscle cells. Am J Physiol Lung Cell Mol Physiol 2014;306:L521-L533.

49 Jha JC, Gray SP, Barit D, et al: Genetic targeting or pharmacologic inhibition of NADPH oxidase Nox4 provides renoprotection in long-term diabetic nephropathy. J Am Soc Nephrol 2014;25:1237-1254.

50 Sedeek M, Callera G, Montezano A, et al: Critical role of Nox4-based NADPH oxidase in glucose-induced oxidative stress in the kidney: implications in type 2 diabetic nephropathy. Am J Physiol Renal Physiol 2010; 299:F1348-F1358.

51 Chen K, Zhang J, Zhang W, et al: ATP-P2X4 signaling mediates NLRP3 inflammasome activation: a novel pathway of diabetic nephropathy. Int J Biochem Cell Biol 2013;45:932943 
52 Wang C, Pan Y, Zhang QY, Wang FM, Kong LD: Quercetin and allopurinol ameliorate kidney injury in STZ-treated rats with regulation of renal NLRP3 inflammasome activation and lipid accumulation. PLoS One 2012; 7:e38285.

53 Bakker PJ, Butter LM, Kors L, et al: Nlrp3 is a key modulator of diet-induced nephropathy and renal cholesterol accumulation. Kidney Int 2014;85:1112-1122.

54 Anders HJ, Schaefer L: Beyond tissue injurydamage-associated molecular patterns, tolllike receptors, and inflammasomes also drive regeneration and fibrosis. J Am Soc Nephrol 2014;25:1387-1400.

55 Gao P, He FF, Tang H, et al: NADPH oxidaseinduced NALP3 inflammasome activation is driven by thioredoxin-interacting protein which contributes to podocyte injury in hyperglycemia. J Diabetes Res 2015;2015: 504761.

56 Gao P, Meng XF, Su H, et al: Thioredoxininteracting protein mediates NALP3 inflammasome activation in podocytes during diabetic nephropathy. Biochim Biophys Acta 2014; 1843:2448-2460.

57 Yang SM, Ka SM, Wu HL, et al: Thrombomodulin domain 1 ameliorates diabetic nephropathy in mice via anti-NF- $\kappa B / N L R P 3$ inflammasome-mediated inflammation, enhancement of NRF2 antioxidant activity and inhibition of apoptosis. Diabetologia 2014;57: 424-434.

58 Kim SM, Lee SH, Kim YG, et al: Hyperuricemia-induced NLRP3 activation of macrophages contributes to the progression of diabetic nephropathy. Am J Physiol Renal Physiol 2015;308:F993-F1003.

59 Shahzad K, Bock F, Dong W, et al: Nlrp3-inflammasome activation in non-myeloid-derived cells aggravates diabetic nephropathy. Kidney Int 2015;87:74-84.

60 Sakai N, Wada T: Revisiting inflammation in diabetic nephropathy: the role of the Nlrp3 inflammasome in glomerular resident cells. Kidney Int 2015;87:12-14.

61 Yi F, Li PL: Mechanisms of homocysteine-induced glomerular injury and sclerosis. Am J Nephrol 2008;28:254-264.

62 Yi F, Zhang AY, Janscha JL, Li PL, Zou AP: Homocysteine activates NADH/NADPH oxidase through ceramide-stimulated Rac GTPase activity in rat mesangial cells. Kidney Int 2004;66:1977-1987.

63 Zhang C, Boini KM, Xia M, et al: Activation of Nod-like receptor protein 3 inflammasomes turns on podocyte injury and glomerular sclerosis in hyperhomocysteinemia. Hypertension 2012;60:154-162.

64 Abais JM, Zhang C, Xia M, et al: NADPH oxidase-mediated triggering of inflammasome activation in mouse podocytes and glomeruli during hyperhomocysteinemia. Antioxid Redox Signal 2013;18:1537-1548.

65 Abais JM, Xia M, Li G, et al: Nod-like receptor protein 3 (NLRP3) inflammasome activation and podocyte injury via thioredoxin-interacting protein (TXNIP) during hyperhomocysteinemia. J Biol Chem 2014;289:2715927168.

66 Krishnan SM, Dowling JK, Ling YH, et al: Inflammasome activity is essential for one kidney/deoxycorticosterone acetate/salt-induced hypertension in mice. Br J Pharmacol 2016;173:752-765.

67 Mulay SR, Evan A, Anders HJ: Molecular mechanisms of crystal-related kidney inflammation and injury. Implications for cholesterol embolism, crystalline nephropathies and kidney stone disease. Nephrol Dial Transplant 2014;29:507-514.

68 Mulay SR, Kulkarni OP, Rupanagudi KV, et al: Calcium oxalate crystals induce renal inflammation by NLRP3-mediated IL- $1 \beta$ secretion. J Clin Invest 2013;123:236-246.

69 Knauf F, Asplin JR, Granja I, et al: NALP3mediated inflammation is a principal cause of progressive renal failure in oxalate nephropathy. Kidney Int 2013;84:895-901.

70 Jakopin Z: Nucleotide-binding oligomerization domain (NOD) inhibitors: a rational approach toward inhibition of NOD signaling pathway. J Med Chem 2014;57:6897-6918. 